Instituto Internacional de Investigación y Desarrollo Tecnológico Educativo INDTEC, C.A.

DOI: https://doi.org/10.29394/Scientific.issn.2542-2987.2018.3.7.19.374-393

OAI-PMH: http://www.indteca.com/ojs/index.php/Revista Scientific/oai

\title{
Estilos de Pensamiento y Enfoques Epistemológicos
}

\author{
Autora: Maryianela del Carmen Maita Guédez \\ Universidad de Los Andes Núcleo Universitario "Dr. Pedro Rincón Gutiérrez", ULA \\ mmaita@ula.ve; maryianelamaita@gmail.com
}

Táchira, Venezuela

\section{Resumen}

Este ensayo inicia con un referente histórico cuyo propósito es distinguir las diversas posturas filosóficas dadas a lo largo de la construcción de la ciencia. El recorrido se divide en dos épocas, antes y después del siglo XVII, por una parte, se enfoca en el pensamiento griego constituido por las tradiciones Aristotélicas y Galileanas; por la otra se presentan las grandes tradiciones epistemológicas: el Racionalismo y el Empirismo. Se continúa en el siglo XIX con Augusto Comte quien introduce el enfoque positivista de la ciencia hasta llegar a la figura de Thomas Kuhn con el concepto de paradigma. Seguidamente, se tratan los estilos de pensamiento y enfoques epistemológicos pues cada ser humano tiene una forma particular de abordar la realidad, aprender, resolver problemas, inferir, desenvolverse en su entorno o seguir ciertos patrones que lo asemeja o diferencia de los otros. Luego se establece la relación entre ambos, demostrándose que son el sustento de cualquier revolución científica exhibida en el transcurso de la historia. Finalmente se concluye que todo trabajo de investigación adquiere un valor en el contexto del sistema de creencias donde ha sido planteado, más que dentro de imposiciones de los paradigmas que establecen esquemas supuestamente indiscutibles y universales.

Palabras clave: epistemología; racionalismo; empirismo; razonamiento. 


\title{
Styles of Thought and Epistemological Approaches
}

\begin{abstract}
This paper begins with a historical reference whose purpose is to distinguish the various philosophical positions given throughout the construction of science. The path is divided into two periods, before and after the seventeenth century, on the one hand, focuses on the Greek thought constituted by the Aristotelian and Galilean traditions; On the other hand are presented the great epistemological traditions: Rationalism and Empiricism. It continues in the XIX with Augusto Comte who introduces the positivist approach to science till the figure of Thomas Kuhn with the concept of paradigm. Then, it is about the styles of thought and epistemological approaches due to each human being has a particular way of approaching reality, learning, solving problems, inferring, developing in their environment or following certain patterns that resemble or differentiate them from others. Then the relationship between them is established, proving that they are the support of any scientific revolution exhibited in the course of history. Finally, it is concluded that all research work acquires a value in the context of the belief system where it has been raised, rather than within impositions of paradigms that establish supposedly indisputable and universal schemes.
\end{abstract}

Keywords: epistemology; rationalism; empiricism; reasoning. 


\section{Introducción}

El conocimiento ha sido, a lo largo de la historia de la ciencia, uno de los grandes debates entre filósofos, en los que se disputan tres creencias: las ontológicas que giran en torno a la naturaleza de los fenómenos, las epistemológicas referidas a las dudas de cómo se puede adquirir, conocer, comunicar el conocimiento y las creencias metodológicas que se concentran en el interés por el modo en que el individuo crea, modifica e interpreta el mundo en el que se encuentra. En otras palabras, dependiendo de cómo se relacione el sujeto que conoce (investigador) con el objeto de estudio, la operación misma de conocer y la información recopilada acerca del objeto, el resultado puede variar de un conocimiento común a un conocimiento científico.

Para Martínez (2010a), el conocimiento es "el resultado de un elaboradísimo proceso de interacción entre un estímulo sensorial (visual, auditivo, olfativo, etc. o un contenido de nuestra memoria) y todo nuestro mundo interno de valores, intereses, creencias, sentimientos, temores, etc." (pág. 176). Por consiguiente, el sujeto que investiga detecta, plantea y busca soluciones a situaciones problemáticas que se presentan en la realidad donde se desenvuelve, manteniendo permanentemente una acción crítica, reflexiva, sensible; como indica Morales (2014), "bajo la actitud de asombro y admiración" (pág. 29), ante el objeto estudiado y los resultados obtenidos.

Según Árraga y Añez (2003a), el individuo común es el mismo que investiga, el cual utiliza sus procesos cognitivos: estilos de aprendizajes (formas de organizar, almacenar información), estilos de pensamientos (formas como perciben, interpretan, construyen la realidad), así como enfoques epistemológicos (postura que adoptan para producir conocimiento científico) que predomina a la hora investigar.

En este ensayo se tratan los estilos de pensamientos, enfoques epistemológicos y la relación entre ambos para demostrar que son el sustento de cualquier revolución científica que se vaya dando en el transcurso de la 
historia. En este sentido se inicia con la conceptualización del término epistemología para luego realizar un recorrido por el pensamiento de algunos autores relevantes de la ciencia, con el propósito de distinguir las variaciones que se han presentado en la forma de concebir y hacer ciencia, abriendo la posibilidad de producir nuevas posturas para la explicación científica que no se fundamenten exclusivamente en lo causal, teleológico o hermenéutico.

\section{La filosofía de las ciencias: en la búsqueda de un punto de encuentro}

La epistemología, o filosofía de la ciencia, para Bunge (2002), "es la rama de la filosofía que estudia la investigación científica y su producto, el conocimiento" (pág. 21), tomando en cuenta los métodos, naturaleza y estructura para obtenerlo, validarlo y socializarlo. Jaramillo (2003), la relaciona con la génesis de los conocimientos científicos, que a través de la objetividad de los mismos se determinan los saberes ideológicos de la época, produciendo un impacto y transformación cultural en las instituciones de una sociedad. Por su parte, Vargas (2015), considera que "da sustento a la reflexión científica de cualquier disciplina" (pág. 208).

De las definiciones anteriores, se puede aseverar que la epistemología es la filosofía de la ciencia, se basa en la reflexión crítica de los fundamentos, principios y demás aspectos que soportan el conocimiento científico (confiable, verificable, validado, socializado, fidedigno, universal) generado en una disciplina para darle el estatus de ciencia, donde se formulan postulados, teorías y leyes que permiten el desarrollo de la humanidad.

En este sentido, la ciencia a lo largo de su construcción ha pasado por diferentes momentos como por diversas posturas filosóficas. Su definición ha estado rodeada de polémicas y variantes, en función de intereses y perspectivas; así lo demuestra Mardones (1991a), en su escrito. Al respecto, al revisar la historia de la concepción de la ciencia, anterior al siglo XVII, se encontrará un predominio de la idea de ciencia heredada del pensamiento 
griego representada por las tradiciones Aristotélicas y Galileanas.

Para Aristóteles (como se cita en Salgado, 2012), la ciencia es:

Conocimiento de lo universal y de las cosas necesarias, y hay unos principios de lo demostrable y de toda ciencia (pues la ciencia es racional), el principio de lo científico no puede ser ni ciencia, ni arte ni prudencia; porque lo científico es demostrable (pág. 3).

Esto implica plena confianza en el poder de la razón, en otras palabras, se llega a la explicación científica, de un fenómeno o hecho, apoyado en la observación del mundo real y la abstracción de la mente humana que de manera ordenada y segura se hacen las demostraciones, por consiguiente, es totalmente contrapuesto a la simple opinión. Según lo argumentado por (Mardones, 1991b), Aristóteles exigía que la causa de un fenómeno debía cubrir cuatros aspectos: la causa formal, la causa material, la causa eficiente y la causa final, haciéndose énfasis en la causa final, revelando explicaciones teleológicas.

Sandín (2003a), documenta que la tradición galileana plantea un nuevo "mundo científico" en la que se rechaza la cosmovisión teleológica de Aristóteles y aparece una visión del mundo principalmente pragmático y mecanicista, en la que la experiencia como fuente de conocimiento tendrá un énfasis mayor. Los precursores de esta postura son Francis Bacon (15611626), quien recoge la propensión de hechos concretos con un intento de dominar la naturaleza. Galileo Galilei (1564-1642), de acuerdo con (Mardones, 1991c), representa "la nueva mentalidad que cambia las explicaciones físicas cualitativas de Aristóteles por las formulaciones matemáticas de Arquímedes" (pág. 25). Consecuentemente, la matemática va a ser el instrumento fundamental para la explicación científica de un fenómeno y cuyas explicaciones tomarán las formas de hipótesis causales que vienen determinada por el análisis experimental. 
Asimismo, (Sandín, 2003b), indica que a partir del siglo XVII surgió el término idealismo para designar la teoría platónica en la que se exponía la posibilidad de que el hombre sólo pudiera conocer "ideas", objetos subjetivos y privados de la mente humana. Desde este siglo hasta la figura de Kant (17241804), con su actitud filosófica denominada Criticismo, los filósofos europeos se circunscriben en dos grandes tradiciones epistemológicas: el Racionalismo en el que figuran pensadores continentales como Descartes (1596-1650), Leibniz (1646-1716) y Spinoza (1632-1677) y el Empirismo representado por autores ingleses como Locke (1632-1704), Hume (1711-1776) y Berkeley (1685-1753).

En la concepción racionalista del conocimiento el saber está constituido por la razón, siendo ésta el único principio y fundamento del conocimiento verdadero, porque solo ésta produce ideas claras, universales e indudablemente ciertas. Así lo afirma Descartes (2010), en su Discurso del Método: "despiertos o dormidos, no debemos dejarnos persuadir nunca sino por la evidencia de la razón" (pág. 65). Usa la matemática como modelo y la intuición intelectual como fuente de conocimiento.

En la tradición empirista el verdadero conocimiento procede de la experiencia, dicho de otra manera, defiende como forma de conocimiento la comprobación detallada de los hechos mediante la observación, son los sentidos, las percepciones y las sensaciones los medios a través de los cuales surgen las ideas en la mente.

El mayor exponente de esta postura Locke (2005a), en el documento original de 1689, asegura que "todas las ideas vienen de la sensación o de la reflexión. Supongamos, entonces, que la mente sea, como se dice, un papel en blanco, limpio de toda inscripción, sin ninguna idea" (pág. 36). Esto significa que la razón humana está vacía antes de recibir la experiencia. Para (Locke, 2005b), existen tres formas elementales de conocimiento correlacionadas con el objeto conocido; Padrón (2014a), los expresa como "la intuición (vivencia, 
introspección, comprensión), la demostración (razonamiento, argumentación, explicación) y la sensación (captación sensorial, observación, instrumentación)" (pág. 1).

Al remontarnos en el siglo XIX, encontramos que Augusto Comte (17981857), introduce el enfoque positivista de la ciencia para designar el conocimiento científico en el que se pretende hacer ciencia social, histórica, económica, entre otras. Comte (2004), en su Curso de Filosofía Positiva publicado de 1830 a 1842, plantea que el primer gran resultado de la filosofía positiva es "la manifestación por la experiencia de las leyes que acompañan en su ejecución a nuestras funciones intelectuales y, por ende, el conocimiento riguroso de las reglas generales convenientes para proceder con seguridad a la búsqueda de la verdad" (pág. 52).

En el enfoque de Comte el verdadero conocimiento científico, que permite descubrir la realidad de lo que se estudia, es el obtenido mediante la observación empírica, la experimentación y la inducción dejando a un lado las concepciones teológicas y metafísicas por no mostrar los hechos tal como son percibidos por los sentidos. En este enfoque la verificabilidad es el criterio para distinguir las ciencias empíricas de otros tipos de conocimientos, fundamentándose en la tradición galileana de la ciencia, siguiendo la tipificación ideal de la física matemática y tratando de enmarcar bajo el mismo método todo saber con pretensiones científicas.

Adicionalmente, el autor en referencia establece como fin de la filosofía positivista el "resumir en un cuerpo de doctrina homogénea la totalidad de los conocimientos adquiridos, relativos a los diferentes órdenes de fenómenos naturales" (pág. 62).

El Circulo de Viena, en las primeras décadas del siglo XX, compuesto por los seguidores del Empirismo de Hume, son los creadores del Positivismo Lógico, también llamado Neopositivismo o Empirismo Lógico. Fijan su interés en introducir, al estudio de la filosofía, métodos y precisión matemática, 
fundamentando las bases que unían verdad y significado, con la finalidad de distinguir entre lo que es ciencia y lo que no lo es. Excluyeron la metafísica, la teoría y la ética del genuino conocimiento humano.

El Positivismo, que imperó por más de tres siglos, no tardó en tener sus detractores, fraguándose en el ámbito alemán una tendencia anti-positivista denominada Hermenéutica, en el que se rechazan las pretensiones del positivismo del monismo metodológico, del afán predictivo y causalista, la reducción de la razón a razón instrumental y la imposición de la físicamatemática como mecanismo de toda explicación científica.

Por su parte, los filósofos de la escuela de Frankfurt creada en 1923, comienzan a cuestionar el Positivismo, estableciendo lo que se denominó posteriormente Teoría Crítica, caracterizada por su conciencia crítica, valga la redundancia, la cual apunta a que las experiencias vividas pueden estar distorsionadas por una falsa ideología o conciencia. Asimismo, según (Martínez, 2010b), los físicos, los psicólogos de la Gestalt, los lingüistas, los biólogos y los filósofos de la ciencia, de la nueva racionalidad científica, mostraron su insatisfacción con la racionalidad lineal y la necesidad de sustituir el modelo axiomático de pensar, razonar y demostrar, donde prima un ideal lógico-matemático, por "una lógica que diera cabida a la auténtica y más empírica realidad del mundo en que vivimos y con el que interactuamos, de un mundo donde existen inconsistencias reales, incoherencias lógicas y hasta contradicciones conceptuales" (pág. 175).

Asimismo, Popper (1902-1994), comienza a prestar atención a la problemática de las ciencias sociales, así queda destacado en su ponencia intitulada La lógica de las ciencias sociales. Popper (1978), manifiesta que el método de éstas radica en ensayar las posibles soluciones a los problemas, pues el conocimiento científico es hipotético y conjetural, en el cual el producto del conocimiento es más un acto de invención que de descubrimiento; se realizan esbozos arbitrarios de las teorías que requieren una comprobación y 
en los casos que no se puede comprobar una hipótesis científica, no se usa la verificación sino la falsabilidad, lo cual debe llevar al científico a rechazarlas. La inducción no constituye una base suficientemente segura para explicar la validez de las teorías científicas, sino que se apoya en la deducción.

La figura de Thomas Kuhn (1922-1996), viene a dar un giro historicista en la filosofía de la ciencia y continuado por Imre Lakatos (1922-1974), para quien el proceso de falsabilidad no parece ni tan simple y lógico como da a entender Popper, por lo que el diseño popperiano de conjeturas y refutaciones ha de ser abandonado, manteniéndose criterios racionales para la sustitución o eliminación de programas de investigación. Kuhn mostraba la inoperancia del criterio popperiano de la falsabilidad. Enseñaba cómo funciona realmente la ciencia, considerándola una actividad organizada, poseedora de ciertos modelos para controlar los resultados, que dependen no sólo de factores lógicos o intelectuales sino también de factores históricos y sociales, prestando atención al proceso mediante el cual se obtiene el conocimiento científico.

Finalmente, cabe destacar que el concepto de paradigma fue generalizado a partir de 1962 en la obra de Kuhn "La Estructura de la Revoluciones Científicas". Para Kuhn (1962), un paradigma "son realizaciones científicas universalmente reconocidas que, durante cierto tiempo, proporcionan modelos de problemas y soluciones a una comunidad científica" (pág. 13); dicho de otro modo, hace referencia a la forma cómo los científicos conciben su área de interés, los problemas a ser estudiados, los métodos a utilizar, entre otros; dentro de su área disciplinar.

\section{Estilos de pensamiento}

Cada ser humano tiene una forma particular de abordar la realidad, aprender, resolver problemas, inferir, desenvolverse en su entorno o seguir ciertos patrones que lo asemeja o diferencia de los otros. Esto se debe primordialmente a los estilos de pensamiento, los cuales se forman antes de 
nacer y se va consolidando en forma gradual en la medida en que el individuo interacciona con su medio ambiente.

Al conceptualizar los estilos de pensamiento, Valadez (2009), revela como aspectos distintivos la manera en que las personas enfocan sus tareas. Dependiendo de las situaciones que se enfrentan se manifestará un estilo determinado, por tanto, existen diferencias en la intensidad del estilo que van de acuerdo a la identificación de la persona con éste. Son estilos variables, en efecto pueden modificarse a lo largo de la vida. En fin, para la autora, el estilo de pensamiento se refiere a una capacidad o aptitud y no a una habilidad.

Por su parte, López y Martín (2010), expresan: "los estilos de pensamiento son los modos en que las personas prefieren utilizar las capacidades intelectuales de que dispone" (pág. 255), es decir se relaciona en cómo se emplea la inteligencia más que en el nivel que se tenga de ésta.

En este ensayo se siguen los planteamientos de (Padrón, 2014b), para quien los estilos de pensamiento representan la personalidad cognitiva, son los responsables del modo en que vemos las cosas, las conocemos y las controlamos. Se orientan hacia tres factores: sentidos (la observación controlada), cerebro (el razonamiento) y corazón (las vivencias e introspecciones).

Sin duda alguna, sentidos, cerebro y corazón coexisten en el individuo, son inseparables, pero en cada circunstancia que se presenta, manejo de información o resolución de problemas uno de ellos tiende a ser el que nos identifica. Al respecto, los estilos de pensamiento son:

- Inductivo - Concreto: los individuos con predominio en este estilo de pensamiento son pragmáticos, se guían principalmente por los sentidos y la observación detallada del mundo que los rodea, ésta es la premisa para comprobar los hechos, razón por la cual requieren el contacto directo con el objeto de estudio. 
- Deductivo - Abstracto: en este caso, los individuos parten de conocimientos generales para mediante la derivación construir nuevos conceptos, la fuente principal para lograrlo es el razonamiento, la argumentación, la deducción. Son teóricos e idealistas, prefieren la información implícita directamente en el objeto de estudio.

- Intuitivo - Vivencial: se fundamentan en el pensamiento, la introspección, la sensibilidad para la búsqueda de soluciones, donde las vivencias internas tienen un gran valor.

\section{Enfoques epistemológicos}

El autor anteriormente citado, documenta los enfoques epistemológicos como conocimiento científico y la vía para producirlo, los cuales son sistemas de convicciones del máximo nivel de profundidad cognitiva, universales, que tienen presencia en la creación del conocimiento científico de la siguiente manera:

- Empirismo: denominado enfoque medicional, se basa en la inducción (experiencia controlada, explicación). Confía en la percepción sensorial. Busca patrones de repetición que se expresan estadísticamente.

- Racionalismo: el enfoque racionalista usa como método de hallazgo la deducción (modelación lógica, explicación). Su principal postulado es la confianza en el razonamiento puro. Persigue estructuras universales básicas amplias.

- Vivencialismo: también llamado enfoque experiencialista, se fundamenta en intuición (experiencia vivida, comprensión). Fija la atención en los simbolismos socioculturales, auxiliada por un instrumento verbalizado de opciones abiertas.

Aunque los enfoques epistemológicos se asocian con la producción de 
conocimiento científico, éstos provienen de los estilos de pensamiento, ambos se producen en el centro holístico privilegiado del ser humano. Sobre esto, (Árraga y Añez, 2003b), afirman que "son de naturaleza cognitiva; es decir, son procesos mentales que tienen lugar en el cerebro" (pág. 36), quien se encarga de procesar la información, gestionarla y resolver problemas.

La relación entre estilos de pensamiento y enfoques epistemológicos viene dada por diversos aspectos, por ejemplo, el foco de atención, las formas de abordar la realidad el canal que se utiliza para el contacto con la realidad, el lenguaje predominante, el objeto de estudio, entre otros.

La tabla 1, muestra las relaciones básicas entre los estilos de pensamiento y los enfoques epistemológicos considerados en este ensayo.

Tabla 1. Relaciones entre los estilos de pensamiento y enfoques epistemológicos.

\begin{tabular}{|c|c|c|c|c|c|c|}
\hline $\begin{array}{c}\text { Estilos de } \\
\text { Pensamiento }\end{array}$ & $\begin{array}{c}\text { Enfoques } \\
\text { Epistemológicos }\end{array}$ & Foco de Atención & $\begin{array}{l}\text { Forma de Abordar } \\
\text { la Realidad }\end{array}$ & Canal & Lenguaje & Objeto de Estudio \\
\hline $\begin{array}{l}\text { Inductivo- } \\
\text { Concreto }\end{array}$ & Empirismo & $\begin{array}{l}\text { Observación } \\
\text { directa de hechos } \\
\text { y objetos } \\
\text { concretos. } \\
\text { Identificación de } \\
\text { patrones de } \\
\text { regularidad o } \\
\text { frecuencia } \\
\text { (Leyes) }\end{array}$ & $\begin{array}{l}\text { Experimentación } \\
\text { concreta. } \\
\text { Observación }\end{array}$ & $\begin{array}{l}\text { Sensorial. } \\
\text { Emotivo }\end{array}$ & $\begin{array}{l}\text { Numérico. } \\
\text { Aritmético }\end{array}$ & $\begin{array}{l}\text { Relaciones } \\
\text { causa-efecto }\end{array}$ \\
\hline $\begin{array}{l}\text { Deductivo- } \\
\text { Abstracto }\end{array}$ & Racionalismo & $\begin{array}{l}\text { Ideas y } \\
\text { conceptos. } \\
\text { Modelación de } \\
\text { procesos } \\
\text { generativos } \\
\text { (Modelos) }\end{array}$ & $\begin{array}{l}\text { Conceptualización } \\
\text { abstracta. } \\
\text { Experimentación } \\
\text { activa }\end{array}$ & Razón & $\begin{array}{l}\text { Verbal. } \\
\text { Lógico- } \\
\text { matemático }\end{array}$ & $\begin{array}{l}\text { Relaciones } \\
\text { generativas }\end{array}$ \\
\hline $\begin{array}{l}\text { Intuitivo- } \\
\text { Vivencial }\end{array}$ & Vivencialismo & $\begin{array}{l}\text { Experimentación } \\
\text { activa de sucesos } \\
\text { internos y } \\
\text { externos. } \\
\text { Intuición. } \\
\text { Construcción } \\
\text { simbólica del } \\
\text { mundo social y } \\
\text { cultural } \\
\text { (Interpretaciones) }\end{array}$ & $\begin{array}{l}\text { Experiencia } \\
\text { concreta. } \\
\text { Experimentación } \\
\text { activa }\end{array}$ & $\begin{array}{l}\text { Kinestésico. } \\
\text { Sensorial }\end{array}$ & $\begin{array}{l}\text { Verbal } \\
\text { académico. } \\
\text { Evocador, } \\
\text { Metafórico }\end{array}$ & $\begin{array}{l}\text { Simbolismos } \\
\text { socioculturales }\end{array}$ \\
\hline
\end{tabular}

Fuente: Adaptación Basada en Árraga y Añez (2003) y Padrón (2014).

Tal como se indicó en el primer apartado, los filósofos de la ciencia se 
han suscrito, según la fuente del conocimiento de una investigación, a dos tradiciones epistemológicas: Racionalismo y Empirismo. Del mismo modo, se presentan dos distinciones ontológicas orientadas a la forma y la naturaleza de la realidad, tomando en cuenta el papel que cumple el sujeto que investiga ante el objeto investigado o realidad estudiada. Estas son Idealismo y Realismo.

En el idealismo la realidad es una idea, se basa en la comprensión e interpretación del objeto de estudio como el resultado del pensar; prevalece la vivencia y la interacción. El realismo, como postura antagónica al idealismo, condiciona el pensar, por tanto, la realidad es independiente de nuestra conciencia, predomina lo que percibimos mediante los sentidos. Se fundamenta en la explicación; mediante la experiencia se llega al conocimiento.

A partir de estos criterios de la relación sujeto-objeto y la fuente de conocimiento, (Padrón, 2014c), considera pueden refinarse 4 enfoques epistemológicos bien diferenciados, con la consolidación de pares conceptuales Empirismo/Racionalismo y Realismo/Idealismo. En la tabla 2 se puede observar cómo se cruzan las distinciones ontológicas y epistemológicas Empirismo - Idealismo, Empirismo - Realismo, Racionalismo - Idealismo, Racionalismo - Realismo, para formar otros enfoques.

Tabla 2. Enfoques epistemológicos.

\begin{tabular}{|c|c|c|}
\hline $\begin{array}{c}\text { Fuente del } \\
\text { Conocimiento }\end{array}$ & \multirow[t]{2}{*}{ EMPIRISMO } & \multirow[t]{2}{*}{ RACIONALISMO } \\
\hline $\begin{array}{c}\text { Relación } \\
\text { Sujeto - Objeto }\end{array}$ & & \\
\hline IDEALISMO & $\begin{array}{l}\text { Enfoque Vivencialista - } \\
\text { Experiencialista } \\
\text { Ejemplos históricos: } \\
\text { Etnografía, etnometodología, } \\
\text { observación participante, } \\
\text { investigación - acción... }\end{array}$ & $\begin{array}{l}\text { Enfoque Vivencialista - } \\
\text { Interpretativista } \\
\text { Ejemplos históricos: Teoría } \\
\text { Crítica, Neodialéctica... }\end{array}$ \\
\hline REALISMO & $\begin{array}{l}\text { Enfoque Empirista } \\
\text { Inductivista } \\
\text { Ejemplos históricos: } \\
\text { Positivismo, medicionismo, } \\
\text { operacionalismo, } \\
\text { instrumentalismo... }\end{array}$ & $\begin{array}{l}\text { Racionalista - Deductivista } \\
\text { Ejemplos históricos: } \\
\text { Racionalismo Cartesiano, } \\
\text { racionalismo crítico, } \\
\text { falsacionismo... }\end{array}$ \\
\hline
\end{tabular}

Fuente: Padrón (2014) 
4.1. Enfoque Vivencialista-Experiencialista: el sujeto, a través del trabajo de campo, tiene un papel protagónico en la obtención de la información sobre lo que desea conocer. De este modo, investigador y objeto de estudio están relacionados de forma interactiva e inevitablemente pueden ser influenciados, en consecuencia, los resultados estarán mediados por los valores del investigador. Habermas (1986), en su trabajo Ciencia y Técnica como Ideología, documento original de 1968, demuestra la imposibilidad de que la ciencia sea una actividad libre de valores e intereses frecuentemente confrontados.

En la intersección, de la tabla 2, Empirismo/ldealismo; la metodología es dialógica y dialéctica, ya que la naturaleza transaccional de la investigación requiere un diálogo entre el sujeto y el objeto estudiado, el cual debe tener una esencia dialéctica para transformar lo desconocido y lo errado en conocimiento, entendiendo las acciones necesarias para efectuar el cambio ante el problema que pretende solucionar. En este enfoque entrarían los trabajos de corte etnográfico, investigación-acción participante, etnometodológicos, diseños de convivencia, entre otros.

4.2. Enfoque Empirista-Inductivista: el investigador confía en la percepción sensorial y persigue patrones de regularidad, a través del trabajo de campo, busca medir, explicar, controlar, predecir. Supone la existencia de una realidad aprehensible impulsada por leyes y mecanismos naturales inalterables. Sujeto y objeto de estudio son entidades independientes, en consecuencia, no deben ser influenciados uno por el otro; alguna influencia, en cualquiera de las dos direcciones, amenaza la validez de la investigación. Adicionalmente, su metodología es experimental y manipuladora en el sentido que las hipótesis o preguntas planteadas se presentan como proposiciones sujetas a una prueba empírica para su verificación. El ejemplo histórico que dominó la ciencia por largo tiempo fue el Positivismo.

4.3. Enfoque Vivencialista-Interpretativista: en este enfoque basado en la 
racionalidad, como fuente de conocimiento, el significado no se descubre, sino que se construye; emerge a partir de la interacción con la realidad y se apoya en experiencias específicas, las ideas o representaciones mentales variadas e intangibles, que dependen de los individuos o grupos que interactúan. Se contrasta con el interpretativismo de manera que se busca comprender y cuestionar al mismo tiempo. Del mismo modo que el enfoque del cruce Empirismo-Idealismo, el investigador y lo investigado están vinculados interactivamente de forma tal que los hallazgos están influenciados, por lo tanto, entra en juego los consensos intersubjetivos y validación activa de quienes producen el conocimiento mediante un intercambio dialéctico. Un ejemplo histórico es la Teoría crítica.

4.4. Enfoque Racionalista-Deductivista: se fundamenta en la razón pura, el acceso al conocimiento, su producción y validación están vinculadas a la construcción de abstracciones que revelan el comportamiento de los hechos (materiales y humanos), los trabajos de campo no se descartan, pero se apoya primordialmente en la interpretación y en los sistemas de razonamiento que van de lo general a lo particular (deducción) con modelaciones lógicomatemáticos. El falsacionismo es uno de los ejemplos históricos de este enfoque.

\section{Reflexiones finales}

En la resolución de problemas de la vida cotidiana y gestión de la información, los estilos de pensamiento son el principal protagonista, ya que son parte inherente al ser humano. Aunque existen diversas clasificaciones de los estilos de pensamiento, aquí se siguió la sugerida por José Padrón, en la que convergen cerebro, sentidos y corazón; que dependiendo de las circunstancias puede predominar uno de los tres estilos: Inductivo - Concreto, Deductivo - Abstracto, Intuitivo - Vivencial.

Ahora bien, en el ámbito de la investigación y la ciencia se habla de 
enfoques epistemológicos los cuales tienen correlación con los estilos de pensamiento, por lo tanto, han existido siempre: desde que el hombre es hombre y la ciencia es ciencia, pues son el sustento de cualquier revolución científica que se ha dado en el transcurso de la historia.

Desde cierto punto de vista, existen al menos tres enfoques epistemológicos amplios y universales, a saber, el enfoque medicional, el enfoque experiencialista y el enfoque racionalista, los cuales se han disputado a lo largo de la historia el control de lo que es o no la ciencia. Sin embargo, haciendo un cruce entre las distinciones ontológicas y las distinciones epistemológicas se refinan cuatro enfoques: Vivencialista - Experiencialista, Empirista - Inductivista, Vivencialista - Interpretativista, Racionalista Deductivista.

El breve recorrido por el pensamiento de algunos autores relevantes de la historia de la ciencia, presentados en este ensayo, nos conduce a señalar que, gracias a los estilos de pensamientos y enfoques epistemológicos, no hay una única teoría que cumpla con todos los requisitos necesarios para hacer ciencia, por el contrario, existen múltiples formas de abordar y solucionar problemas, así como posturas, perspectivas o metodologías.

Cada postura tiene sus seguidores y detractores, pero esto da la posibilidad de producir nuevas alternativas para la explicación científica que no se fundamenten exclusivamente en lo causal, teleológico o hermenéutico sino por el contrario se abren caminos para la complementariedad en beneficio de la obtención de conocimiento científico a través de concreciones metodológicas justificadas que respondan a los requerimientos de credibilidad, confiabilidad y cuestionamiento de los resultados.

Finalmente, ante las controversias dilucidadas, en torno al debate epistemológico, sobre cuáles son las mejores vías y perspectivas para hacer ciencia, es conveniente que en un trabajo de investigación se identifiquen las convicciones donde se sitúa una determinada búsqueda. El definir este marco 
epistemológico, es un elemento clave para analizar y evaluar tanto el proceso de investigación como la representación documentada de los resultados para facilitar la estimación de la calidad de investigación, en virtud de que toda investigación adquiere un valor en el contexto del sistema de creencias en el que ha sido planteado, más que dentro de imposiciones de las revoluciones científicas (Paradigmas) que establecen esquemas supuestamente indiscutibles y universales.

\section{Referencias}

Árraga, M. \& Añez, A. (2003a,b). Aprendizaje, enfoques epistemológicos y estilos de pensamiento. Encuentro Educacional. Vol. 10. № 1 , págs. 23-37. Recuperado de:

http://www.produccioncientificaluz.org/index.php/encuentro/article/view File/907/909

Bunge, M. (2002). Epistemología. Curso de actualización. México D.F, México: Siglo XXI Editores.

Comte, A. (2004). Curso de filosofía positiva. Lecciones 1 y 2. Discurso sobre el espíritu positivo. Recuperado de:

https://es.scribd.com/doc/81133541/Curso-de-Filosofia-Positiva-Comte

Descartes, R. (2010). Discurso del Método. Recuperado de:

http://www.posgrado.unam.mx/musica/lecturas/Lecturalntroduccionlnv estigacionMusical/epistemologia/Descartes-Discurso-Del-Metodo.pdf

Habermas, J. (1986). Ciencia y técnica como ideología. Recuperado de: http://webdelprofesor.ula.ve/ciencias/ricardo/PDF/Jurgen Habermas Ciencia y Tecnica como ideologia.pdf

Jaramillo, L. (2003). ¿Qué es Epistemología? Mi mirar epistemológico y el progreso de la ciencia. Cinta Moebio 18, págs. 174-178. [Documento en línea]. Recuperado de: http://www.revistas.uchile.cl/index.php/CDM/article/viewFile/26135/274 


\section{$\underline{33}$}

Kuhn, T. (1962). La estructura de las revoluciones científicas. Breviarios. Fondo de Cultura Económica.

Locke, J. (2005). Filosofía de John Locke. Ensayo sobre el entendimiento humano. Recuperado de:

https://getafe.es/wp-content/uploads/Locke John-

Ensayo sobre el entendimiento humano.pdf

López, O. \& Martín, R. (2010). Estilos de pensamiento y creatividad. Anales de Psicología, vol. 26, núm. 2, págs. 254-258. Universidad de Murcia, España. Recuperado de:

http://www.redalyc.org/pdf/167/16713079008.pdf

Mardones, J. (1991a,b,c). Filosofía de las ciencias humanas y sociales. Nota histórica de una polémica incesante. Universidad Nacional Abierta. Barcelona: Anthropos Promat. Recuperado de:

http://dip.una.edu.ve/mead/filosofia/paginas/mardones1.pdf

Martínez, M. (2010a,b). Bases de la Epistemología a Comienzos del Siglo XXI. Foundations of Epistemology in Early XXI Century. Revista IIPSI, Facultad de Psicología UNMSM. vol. 13 núm. 1. Recuperado de:

http://sisbib.unmsm.edu.pe/bvrevistas/investigacion psicologia/v13 n1 /pdf/a11.pdf

Morales, J. (2014). Propuesta de una filosofía de las ciencias administrativas y gerenciales desde la praxis y cotidianidad del gerente venezolano en su contexto. (Tesis doctoral, Universidad de Carabobo, Valencia, Venezuela). Recuperado de:

http://mriuc.bc.uc.edu.ve/bitstream/handle/123456789/579/jmorales.pd f?sequence $=4$

Padrón, J. (2014a,b,c). Notas sobre enfoques epistemológicos, estilos de pensamiento y paradigmas. Recuperado de: https://doi.org/10.13140/RG.2.2.22714.08642 
Popper, K. (1978). La Lógica de las Ciencias Sociales. Recuperado de: http://epistemologiadelascienciassociales.sociales.uba.ar/files/2013/04 LLa 1\%C3\%B3gica de las ciencias sociales Popper E A FFyL.pdf Salgado, S. (2012). La filosofía de Aristóteles. Cuadernos Duererías, Serie Historia de la Filosofía núm. 12, ISSN: 1989-7774. Recuperado de: http://guindo.pntic.mec.es/ ssag0007/filosofica/aristotelesduererias.pdf

Sandín, M. (2003a,b). Investigación Cualitativa en Educación. Fundamentos y Tradiciones. Madrid, España: McGraw-Hill.

Valadez, M. (2009). Estilos de aprendizaje y estilos de pensamiento: precisiones conceptuales. Revista de Educación y Desarrollo, núm. 11, págs. 19-30. Recuperado de:

http://www.cucs.udg.mx/revistas/edu desarrollo/anteriores/11/011 Hui zar.pdf

Vargas, J. (2015). Enfoque dialógico a la epistemología de las Ciencias Administrativas. Revista Debates, Porto Alegre. vol. 9. núm. 1, págs. 205-222. Recuperado de: http://www.seer.ufrgs.br/debates/article/viewFile/50173/33508 


\section{Maryianela del Carmen Maita Guédez}

e-mail: mmaita@ula.ve; maryianelamaita@gmail.com

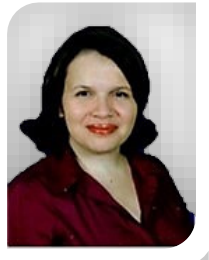

Nacida en el estado Barinas, Venezuela. Licenciada en Educación Mención Informática y Matemática egresada de la Universidad Católica del Táchira (1996). Tiene una Maestría en Matemática mención Educación Matemática de la Universidad Nacional Experimental del Táchira (2005). Doctoranda en Ciencias Gerenciales de la UNEFA Táchira. Trabaja como profesora en la Categoría Asociado de la Universidad de Los Andes Núcleo Universitario "Dr. Pedro Rincón Gutiérrez" Táchira, en el departamento Computación e Informática. Ha publicado su experiencia docente y de investigación en diversas revistas arbitradas. Actualmente acreditada en el Programa de Estímulo a la Innovación e Investigación (PEII).

El contenido de este manuscrito se difunde bajo una Licencia de Creative Commons ReconocimientoNoComercial-Compartirlgual 4.0 Internacional 